\title{
Quick Computation of [C] and [L] Matrices of Generalized Multiconductor Coplanar Waveguide Transmission Lines
}

\author{
Enrique Drake, Francisco Medina, and Manuel Horno, Member, IEEE
}

\begin{abstract}
An enhanced spectral domain quasi-TEM analysis of generalized coplanar waveguide transmission lines (GCPWTL) is presented. The analysis starts from the formulation of a convolution-type integral equation for the electric field at the slots. Chebyshev polynomials including Maxwell singularities are used as basis functions to solve the integral equation by the Galerkin method. Fast and accurate quasi-analytical formulas are used to calculate the Galerkin's matrix entries, thereby significantly reducing the involved CPU time and increasing reliability and accuracy. These features make this technique useful and competitive as CAD tool for coplanar waveguide designs.
\end{abstract}

\section{INTRODUCTION}

C OPLANAR waveguide (CPW) transmission lines are becoming a competitive alternative to microstrip in many applications (including both hybrid and monolithic technologies). A number of attractive features-location of the signal grounds on the same substrate surface as the signal line, low parasitic inductances, easy shunt and series connections, avoidance for the need of via holes, good isolation in directional couplers, etc. [1]-[3]-makes this transmission medium particularly interesting. Due to this fact together with the relative lack of design data available for CPW structures (in comparison with the microstrip line), research on many aspects related to the characterization of $\mathrm{CPW}$ structures is still going on [4]-17].

The computation of the propagation characteristics of CPWs has received some attention in old and recent literature (see, for example, [8]-[11], which include exhaustive bibliography sweeping a wide variety of analytical and numerical techniques). As it is well known, the evaluation of dispersion, radiation, higher-order modes or leakage phenomena requires rigorous hybrid-mode approaches. However, the quasi-TEM approximation can be expected to yield useful results in the frequency band whereon MIC's usually operate today, at least for those structures and components which are not particularly frequency-sensitive [8], and even up to $40 \mathrm{GHz}$ in the design of coplanar MMIC's [9]. Since the quasi-TEM analysis requires

Manuscript received November 17, 1993; revised January 27, 1994 This work was supported in part by the DGICYT, Spain, under contract TIC91-1018.

The authors are with the Microwaves Group. Department of Electronic and Electromagnetism. Facultad de Física Avda. Reina Mercedes s/n, 41012 Sevilla, Spain

IEEE Log Number 9405369 much less computational effort, it is more adequate for design purposes. In addition, quasi-TEM data could eventually be used as initial guesses in full-wave algorithms, thus improving their efficiency.

The quasi-TEM analysis of certain particular CPW geometries has been already carried out in a very efficient way (suitable for CAD applications). For instance, a quasianalytical method to deal with a single CPW embedded in a stratified medium is reported in [11]. In that paper the reader can find a list of references reporting other quasianalytical methods to analyze a variety of symmetrical and asymmetrical single CPW geometries (most of them based on conformal mapping approaches). One of the most recent contributions based on conformal mapping can be found in [6]. However, more complex CPW structures involving multiple dielectric layers and coupled conductors have received less attention in spite of its obvious interest in practical applications (filter, couplers, etc.). Some analytical or approximate solutions for symmetrical coupled structures have been reported in the literature [1], [12]. More sophisticated geometries have been considered in [13], [14]. A recent work [15] proposes an efficient Wiener-Hopf solution for a multiconductor CPW system (for application as interdigital transducer), but it is restricted to geometries symmetrically placed between two ground planes with homogeneous medium. The availability of quick and versatile multiconductor solvers is important from the designer's perspective, since optimization processes involve the iterative evaluation of the parameters of a structure for a wide range of design variables. In this sense, extremely efficient algorithms have been already developed for the quasi-TEM analysis of general multistrip geometries [16]-[18]. However, as far as we know, a systematic and quasi-analytical treatment of a generalized coplanar waveguide transmission line (GCPWTL) system-including an arbitrary number of metallic strips between two ground planes embedded in a multilayer medium-has not been explicitly given yet. The current paper contributes to the computer-aided design of coplanartype circuits by offering a quasi-analytical procedure to compute the quasi-TEM propagation parameters of the GCPWTL system in Fig. 1. The multislot geometry of the GCPWTL system makes suitable to state the analysis in terms of the aperture electric field. Therefore, a convolution integral equation is proposed to connect the electric field at the slots with the free charge on the metallizations. The 


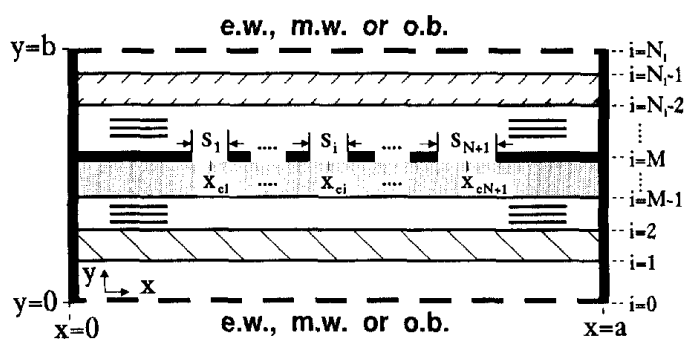

Fig. 1. Cross-section of the generalized multiconductor coplanar waveguide transmission line (GCPWTL).

method used to solve the electric field integral equation is an enhanced Galerkin spectral domain analysis (SDA). Quasianalytical formulas are provided to compute the Galerkin's matrix entries. A dual treatment of multistrip geometries in terms of the free charge density on the metallizations was successfully used and reported in [17]. High speed of computation and extreme accuracy could make the use of this method useful in the CAD of coplanar waveguide circuits.

\section{OUtLINE OF THE Problem}

Fig. 1 shows the cross-section of the GCPWTL analyzed in this work. Translational symmetry in the propagation direction ( $z$-axis) is assumed. The whole structure is enclosed into a rectangular box defined by the planes $x=0, x=a, y=0$, and $y=b$. The lateral planes $x=0$ and $x=a$ are electric walls (e.w.). The planes $y=0$ and $y=b$ can be chosen to be electric walls, magnetic walls (m.w.) or open boundaries (o.b.). The substrate is a $N_{l}$-layered lossless/lossy iso/anisotropic linear medium. An arbitrary number $(N)$ of conductor strips-allocated between two grounded metal fins-are printed on the $M$ th interface. Let us characterize each of the $N+1$ slots between these conductor strips by both its width $\left(s_{i} ; i=1, \ldots, N+1\right)$ and the position $\left(x_{c i}\right.$; $i=1, \ldots, N+1$ ) of its middle point (with respect to the left lateral wall). This structure includes a large group of CPW geometries as particular cases. Moreover, the configuration in Fig. 1 accounts for possible technological constraints (upper and lateral shielding, conductor backing, and line-to-line coupling).

As is well known, all the quasi-TEM propagation parameters of a $\mathrm{N}$-conductor transmission line may be obtained from its capacitance, $[C]$, and inductance, $[L]$, per unit length (p.u.1.) matrices. The determination of $[C]$ implies to solve an electrostatic problem. $[L]$ may be computed from the capacitance, $\left[C^{\prime}\right]$, of a related structure [19]. If the electrostatic problem is stated in terms of the surface free charge density on the conductors, each coefficient, $C_{i j}$, of $[C]$ or $\left[C^{\prime}\right]$ is identified as the free charge on the $i$-th conductor when the $j$-th conductor is set to voltage unity and the rest of the conductors are grounded (canonical voltage excitation) [17]. However, the multislot geometry is more efficiently characterized in terms of the aperture electric fields than in terms of the charge distribution. Owing to this, it is more direct to calculate its coefficients of potential matrix $[P]$, i.e., the set of coefficients, $P_{i j}$, which linearly relates the potential $V_{i}$ of any conductor to the free charges $Q_{j}$ on all of the conductors, including itself:

$$
V_{i}=\sum_{j=1}^{N} P_{i j} Q_{j} \quad(i=1, \ldots, N) .
$$

Note that $[P]=[C]^{-1}$. Each coefficient $P_{i j}$ may be defined as the voltage of the $i$ th conducting strip when the $j$-th strip is charged with charge unity, and the rest of the strips are discharged (canonical charge excitation). The voltage of each strip is computed integrating the electric field $x$-component along the slots existing between one of the grounded plates and that strip. Therefore, $[P]$ (and its inverse, $[C]$ ) will be obtained if we compute the aperture fields for $N$ independent canonical charge excitations.

\section{THE SLOT Electric FiELd EOUation}

From the Green's theorem, the electrostatic potential $\Phi(x)$ on the metallized interface of the structure in Fig. 1 is related to the free charge density $\sigma(x)$ by

$$
\Phi(x)=\int_{0}^{a} G\left(x, x^{\prime}\right) \sigma\left(x^{\prime}\right) d x^{\prime}
$$

where $G\left(x, x^{\prime}\right)$ is the potential Green's function associated to the $M$ th interface of the structure. This is the free charge density integral equation usually solved when multistrip geometries are analyzed. However, we are now interested in using an integral equation for the slot electric field $x$-component. Owing to the existence of electric walls in both $x=0$ and $x=a$, the Fourier series expansion of $G\left(x, x^{\prime}\right)$ yields

$$
G\left(x, x^{\prime}\right)=\frac{2}{a} \sum_{n=1}^{\infty} \widetilde{G}_{0}\left(\alpha_{n}\right) \sin \left(\alpha_{n} x\right) \sin \left(\alpha_{n} x^{\prime}\right) ; \quad \alpha_{n}=\frac{n \pi}{a}
$$

where-from Fourier transform theory $[20]-\widetilde{G}_{o}(\alpha)$ is the spectral domain Green's function (SDGF) associated to the laterally open structure, i.e., $a \rightarrow \infty$. Defining the following pairs of sine(cosine)-Fourier transforms:

$$
\begin{aligned}
F(x) & =\frac{2}{a} \sum_{n=1}^{\infty} \widetilde{F}\left(\alpha_{n}\right)\left\{\begin{array}{l}
\sin \\
\cos
\end{array}\right\}\left(\alpha_{n} x\right) \rightleftharpoons \widetilde{F}\left(\alpha_{n}\right) \\
& =\int_{0}^{a} F(x)\left\{\begin{array}{l}
\sin \\
\cos
\end{array}\right\}\left(\alpha_{n} x\right) d x
\end{aligned}
$$

and using (3), it is straightforward to convert (2) into

$$
\widetilde{\Phi}\left(\alpha_{n}\right)=\widetilde{G}_{0}\left(\alpha_{n}\right) \widetilde{\sigma}\left(\alpha_{n}\right)
$$

by sine-Fourier transform. Simple manipulations let us to rewrite $(5)$ in the following way:

$$
\left(-\frac{\tilde{\sigma}\left(\alpha_{n}\right)}{\alpha_{n}}\right)=\tilde{\mathcal{L}}\left(\alpha_{n}\right)\left(-\alpha_{n} \tilde{\Phi}\left(\alpha_{n}\right)\right)
$$


where

$$
\widetilde{\mathcal{L}}\left(\alpha_{n}\right)=\frac{\widetilde{G}_{0}\left(\alpha_{n}\right)^{-1}}{\alpha_{n}^{2}}
$$

Note that (6) may be identified as the cosine-Fourier transform of

$$
q(x)=\int_{0}^{a} \mathcal{L}\left(x, x^{\prime}\right) E_{x}\left(x^{\prime}\right) d x^{\prime}
$$

where $E_{x}^{\prime}\left(x^{\prime}\right)$ is the electric field $x$-component along the metallized interface. $q(x)$ in (8) is the amount of free charge allocated from $x^{\prime}=0$ to $x^{\prime}=x$, i.e.,

$$
q(x)=\int_{0}^{x} \sigma\left(x^{\prime}\right) d x^{\prime}
$$

and $\mathcal{L}\left(x, x^{\prime}\right)$ is

$$
\mathcal{L}\left(x, x^{\prime}\right)=\frac{2}{a} \sum_{n=1}^{\infty} \tilde{\mathcal{L}}\left(\alpha_{n}\right) \cos \left(\alpha_{n} x\right) \cos \left(\alpha_{n} x^{\prime}\right) .
$$

It is obvious that $q(x)$ stands constant along each slot $(x \in$ $S_{i} \equiv\left[x_{c i}-s_{i} / 2 \leq x \leq x_{c i}+s_{i} / 2\right]$ ), and its value is

$$
q\left(x \in S_{i}\right)=\sum_{j=0}^{i-1} Q_{j} \quad i=1, \ldots, N+1
$$

where $Q_{j} \quad(j=1, \ldots, N)$ is the total free charge on the $j$-th strip, and $Q_{0}$, the total free charge on the left coplanar grounded fin. Since $Q_{0}$ is not known, the total value of $q(x)$ along a slot can not be computed when a charge excitation is imposed. However, when $x$ skips from a given slot to the next one, the increase in $q(x)$ is equal to the amount of free charge supported by the strip allocated between the two slots. Therefore, the electric field $x$-component for the $k$ th $(k=1, \ldots, N)$ canonical charge excitation (charge unity on the $k$ th strip keeping the rest of the strips discharged) fulfils the following condition

$$
\begin{aligned}
& \int_{0}^{a} \mathcal{L}\left(x \in S_{i+1}, x^{\prime}\right) E_{x}\left(x^{\prime}\right) d x^{\prime} \\
& \quad-\int_{0}^{a} \mathcal{L}\left(x \in S_{i}, x^{\prime}\right) E_{x}\left(x^{\prime}\right) d x^{\prime}=\delta_{i k} \quad i=1, \ldots, N
\end{aligned}
$$

where $\delta_{i k}$ is the Kronecker delta ( 1 if $i=k, 0$ if $i \neq k$ ). In addition, the grounding of the lateral fins makes the electric field $x$-component fulfils

$$
\int_{0}^{a} E_{x}\left(x^{\prime}\right) d x^{\prime}=0
$$

\section{METHOD OF ANALYSIS}

Probably, one of the best known techniques to solve integral equations as (12) and (13) is the Galerkin method. In this work, this method has been also chosen. The first kind Chebyshev polynomials, $T_{q}(\cdot)$, weighed by the Maxwell edge singularity are used as basis functions in the expansion of the electric field $x$-component

$$
E_{x}\left(x^{\prime}\right)=\sum_{j=1}^{N+1} \sum_{q=0}^{n f_{j}-1} a_{q, j} E_{x_{q, j}}\left(x^{\prime}\right)
$$

$E_{x_{q, j}}\left(x^{\prime}\right)=\left\{\begin{array}{l}\frac{2}{\pi s_{j}}\left[\begin{array}{c}1-\left(\frac{x^{\prime}-x_{c j}}{s_{j} / 2}\right)^{2} \\ 0 ;\end{array}\right]^{-\frac{1}{2}} \cdot T_{q}\left(\frac{x^{\prime}-x_{c j}}{s_{j} / 2}\right) ; x^{\prime} \in S_{j} \\ \text { elsewhere. }\end{array}\right.$

The choice of this set of functions is suggested by the nature of the integral kernel. The application of the Galerkin method converts (12) and (13) into a system of algebraic linear equations for the coefficients $a_{q, j}$

$$
\begin{aligned}
& \sum_{j=1}^{N+1} \sum_{q=0}^{n f_{j}-1}\left[A_{0, i+1}^{q, j}-A_{0, i}^{q, j}\right] a_{q, j}=\delta_{i k} \quad i=1, \ldots, N \\
& \sum_{j=1}^{N+1} \sum_{q=0}^{n f_{j}-1} A_{p, i}^{q, j} a_{q, j}=0 \quad p \neq 0 \quad i=1, \ldots, N+1 \\
& \sum_{j=1}^{N+1} a_{0, j}=0
\end{aligned}
$$

where the entries $A_{p, i}^{q, j}\left(p=0, \ldots, n f_{i}-1 ; q=0, \ldots, n f_{j}-1\right.$; $i, j=1, \ldots, N+1$ ) of the system are:

$$
\begin{aligned}
A_{p, i}^{q, j}= & \int_{x_{c i}-s_{i} / 2}^{x_{c i}+s_{i} / 2} d x E_{x_{p, i}}(x) \int_{x_{c j}-s_{, j} / 2}^{x_{c j}+s_{j} / 2} d x^{\prime} \\
& \times E_{x_{q, j}}\left(x^{\prime}\right) \mathcal{L}\left(x, x^{\prime}\right) .
\end{aligned}
$$

However $\mathcal{L}\left(x, x^{\prime}\right)$ in (17) is not known (except for special cases) in closed form. Fortunately, (7) shows that the spectral transform, $\widetilde{\mathcal{L}}(\alpha)$, of the kernel of $(17)$ is related to the SDGF, $\widetilde{G}_{0}(\alpha)$, associated to the laterally open version of the multilayered configuration. Therefore, it is more suitable to obtain a spectral domain expression for the entries $A_{p, i}^{q, j}$, and, then, take advantage from the efficient algorithm reported in [19], [21] for computing the SDGF of an arbitrary layered configuration. The spectral version of (17) may be deduced from Parseval and convolution theorems

$$
A_{p, i}^{q, j}=\frac{2}{a} \sum_{n=1}^{\infty} \widetilde{E}_{x_{p, i}}^{*}\left(\alpha_{n}\right) \cdot \widetilde{\mathcal{L}}\left(\alpha_{n}\right) \cdot \widetilde{E}_{x_{q, 3}}\left(\alpha_{n}\right)
$$

where $\widetilde{E}_{x_{q, j}}\left(\alpha_{n}\right)$ are the cosine-Fourier transforms of $E_{x_{q, j}}\left(x^{\prime}\right)$, i.e.,

$\widetilde{E}_{x_{q, j}}\left(\alpha_{n}\right)=\left\{\begin{array}{cc}J_{q}\left(\frac{\alpha_{n} s_{j}}{2}\right)(-1)^{\frac{q}{2}} \cos \left(\alpha_{n} x_{c j}\right) & \text { if } q \text { is even } \\ J_{q}\left(\frac{\alpha_{n} s_{j}}{2}\right)(-1)^{\frac{1(q+1)}{2}} \sin \left(\alpha_{n} x_{c j}\right) & \text { if } q \text { is odd }\end{array}\right.$

with $J_{q}(\cdot)$ being the first kind Bessel function of order $q$.

Once the system (16) has been solved, the coefficients of potential are directly computed from the expansion zerothorder coefficients

$$
P_{i k}=-\sum_{j=1}^{i} a_{0, j} \quad i=1, \ldots, N \quad k \text { th } \quad \text { excitation }
$$




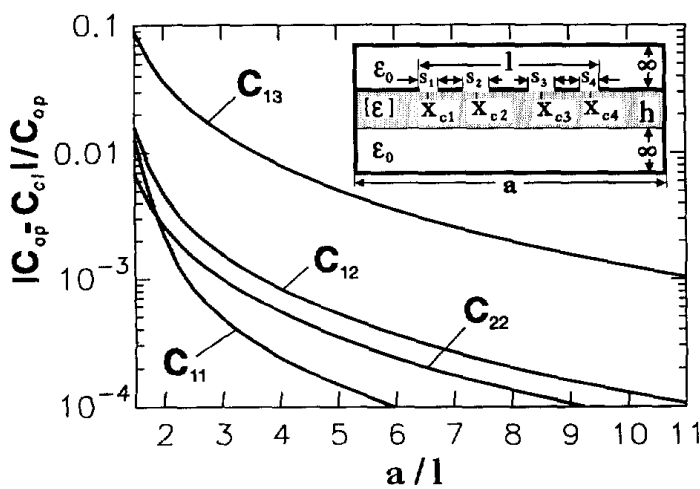

Fig. 2. Relative differences between the capacitance coefficients of a laterally closed three-conductor CPW-type transmission line $\left(C_{\mathrm{cl}}\right)$ and its corresponding open version $\left(C_{o p}\right)$. Data: $h=0.635 \mathrm{~mm}, l=2.4 \mathrm{~mm}$, $s_{1}=s_{2}=s_{3}=s_{4}=0.2 \mathrm{~mm}, x_{\mathrm{c} 1}=a / 2-1.1 \mathrm{~mm}, x_{\mathrm{c} 2}=a / 2-0.5 \mathrm{~mm}$ $x_{c 3}=a / 2+0.5 \mathrm{~mm}, x_{c 4}=a / 2+1.1 \mathrm{~mm}, \epsilon_{x x}=13 \epsilon_{0}, \epsilon_{y y}=10 \epsilon_{0}$.

since only the zeroth-order basis functions in (15) have nonvanishing integrals along their definition intervals.

The computational step of the determination of $[P]$ involving most of the CPU time is precisely the sum of the spectral series in (18). Its direct sum is not advisable because of its very slow convergence. In this work, the Kummer's method (extraction of an asymptotic tail) is used to improve the series convergence. The series of (18) are then split as follows:

$$
\begin{gathered}
A_{p, i}^{q, j}=\frac{2}{a}\left\{\sum_{n=1}^{\infty} \widetilde{E}_{x_{p, i}}^{*}\left[\widetilde{\mathcal{L}}-\widetilde{\mathcal{L}}_{a s}\right] \widetilde{E}_{x_{q, j}}+S_{p, i}^{q, j}\right\} \\
S_{p, i}^{q, j}=\sum_{n=1}^{\infty} \widetilde{E}_{x_{p, i}}^{*} \widetilde{\mathcal{L}}_{a s} \widetilde{E}_{x_{q, j}} ; \widetilde{\mathcal{L}}_{a s}=\frac{2 \bar{\epsilon}}{\alpha_{n}} ; \bar{\epsilon}=\frac{\epsilon_{M}+\epsilon_{M+1}}{2}
\end{gathered}
$$

where $\epsilon_{k}(k=M, M+1)$ is the permittivity (or the equivalent permittivity [19], [21] in the anisotropic case) of the $k$-th layer. Since $\widetilde{\mathcal{L}}_{a s}$ has the same asymptotic behavior than $\widetilde{\mathcal{L}}$ for large $\alpha_{n}$, the remainder series (first term at the right hand of (21)) converges very quickly. The asymptotic tails $S_{p, i}^{q, j}$ are extremely slow convergent series, but quasianalytical expressions for them are provided in Appendix. The methods employed to obtain the formulas in Appendix have been already described in [17]; thus, we have just included in this Appendix the final formulas which applies to the series involved in the analysis of GCPWTL structures.

\section{NUMERICAL RESULTS}

A FORTRAN program (MULTISLOT) has been developed implementing the theory in this paper. The computer code runs on a $\mathrm{PC} / 486 / 33 \mathrm{MHz}$. In order to validate our method, we have reproduced analytical data obtained by means of exact conformal mappings (which are available for some particular geometries). Good agreement has also been found with data reported for more complicated geometries which were obtained by numerical procedures. An interesting comparison has been carried out with the results reported in [15]. The method used in that paper is inherently very accurate (although, in principle, it is limited to homogeneous or symmetrical geometries). The results reported in [15] are the Fourier transforms of the surface charge distributions at the "active" metallization plane of several SAW (surface acoustic wave) structures for several electrode excitation conditions. We have reproduced their results with very good agreement and very few basis functions and short CPU time (typically less than one second on the afore-mentioned computer platform). Minor discrepancies were detected for large values of the Fourier variable, due to the different nature of the basis functions used in the expansions of the unknown functions (surface charge density or slot electric field). We believe our results are even more accurate since our basis functions incorporate the singular behaviour at the metallic edges and the results do not modify when the number of functions increase above a certain value (numerical stability).

In addition, exhaustive convergence tests have been performed to identify the geometrical dimensionless ratios governing the convergence of our codes. This kind of study increases our confidence in our results. The conclusions from these tests are analogous to the ones reported in [17] for the multistrip case. We can summarize here the main points highlighted by this study:

1) The residual spectral series (first term at the right hand in (21)) show exponential convergence. The main geometrical parameter governing this convergence is the ratio $h / a$ ( $h$ being the thickness of the thinnest layer adjoining the metallized interface and $a$ being the width of the enclosure). Therefore, the parameter $a$ should not be chosen unnecessarily large when laterally open structures have to be simulated, since this could increase the number of Fourier terms to be added. For judicious values of $a$ just a few spectral terms are typically required. An example of the influence of the box width on the capacitance coefficients is shown in Fig. 2. In this figure, the relative difference between the capacitance coefficients of a closed structure and its laterally open version is plotted. Note that from a practical point of view the box width does not need to be very large to simulate the open structure.

It is important to highlight that the width of the slots/strips region (distance between the two coplanar ground planes) has not influence on the convergence of the residual spectral series, thus avoiding the typical convergence problems arising when force brute summation is used to analyze narrow slots/strips regions. These statements are illustrated with the example in Table I. Note the important CPU time savings for all of the geometries.

2) The obtaining of the asymptotic tails, $S_{p, i}^{q, j}$, involves a negligible computational cost in comparison with straightforward Fourier series summation. Nevertheless, it is useful to know which factor(s) may affect to the convergence of the power series or Gauss-Chebyshev quadratures shown 
TABLE I

Nlmeer of Terms of the SPectral Series with $\left(n_{r}\right)$ and Without $\left(n_{s}\right)$ Asymptotic Extraction Which Should Be AdDEd to Obtain the Capacitance of a CPW with $0.1 \%$ Accuracy $\left(s_{1}=s_{2}=w, \epsilon=9.9 \epsilon_{0}\right)$. RATIO OF CPU TIMES $\left(t_{r} / t_{s}\right)$

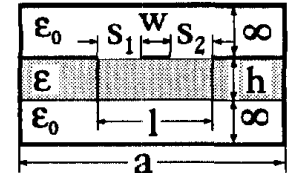

\begin{tabular}{|c|c|c|c|c|}
\hline$l / a$ & $h / a$ & $n_{r}$ & $n_{s}$ & $t_{r} / t_{s}$ \\
\hline 0.01 & 0.2 & 0 & 28000 & 0.005 \\
0.05 & 0.2 & 1 & 5500 & 0.025 \\
0.10 & 0.2 & 3 & 2900 & 0.046 \\
0.25 & 0.2 & 3 & 1500 & 0.088 \\
0.50 & 0.2 & 3 & 700 & 0.188 \\
\hline 0.25 & 1.00 & 0 & 1500 & 0.086 \\
0.25 & 0.30 & 3 & 1500 & 0.086 \\
0.25 & 0.10 & 7 & 1500 & 0.086 \\
0.25 & 0.03 & 15 & 1500 & 0.100 \\
0.25 & 0.01 & 37 & 1500 & 0.127 \\
\hline
\end{tabular}

in the Appendix. The efficiency of the computation of the tails is essentially controlled by the relative proximity between the slots, in such a way that when very tightly coupled slots (very narrow strips) are present more terms must be retained to add up the power series (24) and more quadrature points are necessary in Gauss-Chebyshev integrations (28). Nevertheless, very few power series terms are required except for impracticable strip width, and the number of quadrature points do not need to be larger than the number of basis functions (although it can eventually be increased to account for extremely narrow strips). In any case, asymptotic extraction is always advisable, since direct summation has always much worse numerical performance.

3) The number of basis functions, $n f_{i} ; i=1, \ldots, N+1$, to be retained over each slot is related to its width. Thanks to the appropriate features of the basis functions, no more than two or three of them have to be used on each slot in most cases. A typical convergence pattern is shown in Table II. An interesting point to be emphasized here is that when the number of basis functions is increased, no numerical problems arise. On the contrary we have found numerical instabilities when direct summation of Fourier series is applied. As an additional advantage of our procedure, we can say that the expansion coefficients in (14) are computed with extreme accuracy. The slot electric field is then obtained in addition to the electrical parameters. This is very difficult with force brute summation unless a prohibitively large number of Fourier terms is retained.

In order to check the results of our computer program when applied to arbitrary multislot geometries-for which we have not found published data-we have compared with results generated with a program (MULTISTRIP) written to efficiently analyze multistrip structures [17]. When this code is used,
TABLE II

Convergence of the Capacitance Coefficients (Normalized to fo) with the Number of Basis Functions at each Slot. Data: The Strlcture in

FIG. 2 WITH $a=40 \mathrm{~mm}, h=0.635 \mathrm{~mm}, l=5.2 \mathrm{~mm}, s_{1}=s_{4}=1.0 \mathrm{~mm}$, $s_{2}=s_{3}=0.1 \mathrm{~mm}, x_{c 1}=17.9 \mathrm{~mm}, x_{c 2}=18.95 \mathrm{~mm}, x_{c 3}=21.05$; $\mathrm{mm}, x_{c 4}=22.1 \mathrm{~mm}, \epsilon_{x x}=13 \epsilon_{0}, \epsilon_{y y}=10 \epsilon_{0}, n f_{3}=n f_{2}, n f_{4}=n f_{1}$

\begin{tabular}{|c|c|c|c|c|c|}
\hline$n f_{1}$ & $n f_{2}$ & $C_{11} / \epsilon_{0}$ & $C_{12} / \epsilon_{0}$ & $C_{13} / \epsilon_{0}$ & $C_{22} / \epsilon_{0}$ \\
\hline 1 & 1 & 18.041 & -13.096 & -0.139 & 29.643 \\
2 & 2 & 17.830 & -12.907 & -0.127 & 29.303 \\
3 & 2 & 17.824 & -12.897 & -0.126 & 29.266 \\
4 & 2 & 17.823 & -12.896 & -0.126 & 29.264 \\
5 & 2 & 17.823 & -12.896 & -0.126 & 29.264 \\
\hline
\end{tabular}

TABLE III

CapactTance Coefficients for the Equivalent Mlltistrip Transmission Line (MSTL) and Coplanar Waveguide Transmission LiNe (CPWTL) Geometries Shown in (A) AND (B). DATA: $a=20 \mathrm{~mm}, w_{1}=w_{3}=0.5 \mathrm{~mm}$, $w_{2}=1.0 ; \mathrm{mm}, \mathrm{s}=0.2 \mathrm{~mm}, h=0.635 \mathrm{~mm}, \mathrm{f}=9.6 \mathrm{~F}_{0}$
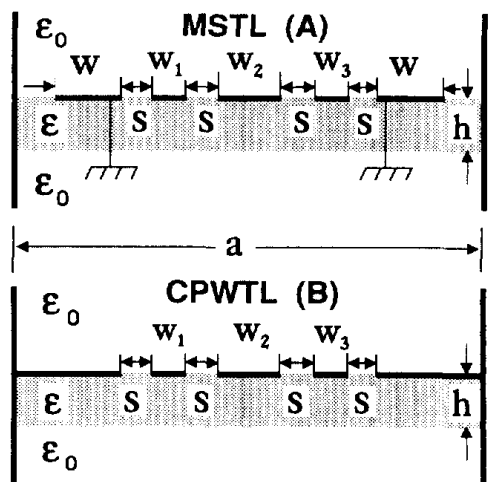

\begin{tabular}{|c|c|c|c|c|}
\hline \multicolumn{5}{|c|}{ Capacitance coefficients for structure A } \\
\hline$w(m m)$ & $C_{11} / \epsilon_{0}$ & $C_{12} / \epsilon_{0}$ & $C_{13} / \epsilon_{0}$ & $C_{22} / \epsilon_{0}$ \\
\hline 1 & 16.6012 & -7.7547 & -0.3047 & 18.8414 \\
2 & 16.6194 & -7.7441 & -0.2999 & 18.8513 \\
3 & 16.6206 & -7.7428 & -0.2992 & 18.8530 \\
4 & 16.6208 & -7.7425 & -0.2991 & 18.8535 \\
\hline \multicolumn{5}{|c|}{ Capacitance coefficients for structure B } \\
\hline$\infty$ & 16.6210 & -7.7423 & -0.2990 & 18.8537 \\
\hline
\end{tabular}

the GCPW geometry is simulated by using wide grounded strips to approximately account for lateral ground planes. Table III shows the capacitance coefficients of a three strips CPW structure when computed with MULTISLOT and when computed with MULTISTRIP. In the last case, the original structure is simulated with a five strip configuration with grounded extreme strips. The results of this simulation for several values of the widths of the extreme strips are shown in Table III. Consistent results for the capacitance parameters have been found with both computer codes. However, the CPU time used by MULTISTRIP is ten times the CPU time used by MULTISLOT $(0.12$ seconds in a PC/486/33 $\mathrm{MHz}$ computer, including the computations for the structure in vacuum). This difference is due to the following fact: for this type of geometries the number of basis functions re- 
TABLE IV

Capacitance Coefficients of the Six Strips Coplanar Waveguide Type Geometry Shown dn the Figure. The Plane aA ${ }^{\prime}$ Is A. Symmetry Plane. Data: $a=20 \mathrm{~mm}, w_{1}=0.4 \mathrm{~mm}$, $w_{2}=0.6 \mathrm{~mm}, w_{3}=0.8 \mathrm{~mm}, s_{1}=s_{4}=0.3 \mathrm{~mm}, s_{2}=0.2 \mathrm{~mm}$, $s_{3}=0.1 \mathrm{~mm}, h_{1}=0.2 \mathrm{~mm}, h_{2}=0.2 \mathrm{~mm}, \epsilon_{1}=9.6 \epsilon_{0}, \epsilon_{2}=4.0 \epsilon_{0}$

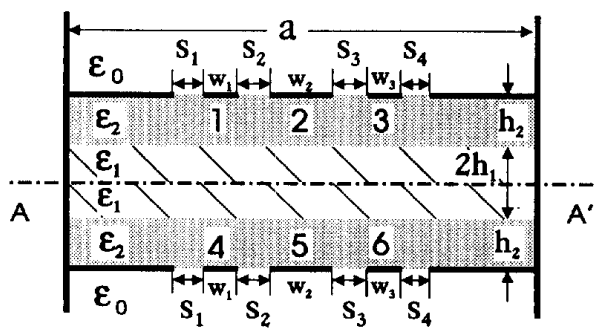

\begin{tabular}{|lr|l}
\hline$C_{11} / \epsilon_{0}=C_{44} / \epsilon_{0}=9.2201$ & $C_{22} / \epsilon_{0}=C_{55} / \epsilon_{0}=12.1982$ \\
$C_{12} / \epsilon_{0}=C_{45} / \epsilon_{0}=-2.5060$ & $C_{23} / \epsilon_{0}=C_{56} / \epsilon_{0}=-3.8203$ \\
$C_{13} / \epsilon_{0}=C_{46} / \epsilon_{0}=-0.2496$ & $C_{25} / \epsilon_{0}=$ & -2.5589 \\
$C_{14} / \epsilon_{0}=$ & -1.7894 & $C_{26} / \epsilon_{0}=C_{35} / \epsilon_{0}=-1.2007$ \\
$C_{15} / \epsilon_{0}=C_{24} / \epsilon_{0}=-0.9947$ & $C_{33} / \epsilon_{0}=C_{66} / \epsilon_{0}=13.5831$ \\
$C_{16} / \epsilon_{0}=C_{34} / \epsilon_{0}=-0.1632$ & $C_{36} / \epsilon_{0}=$ & -3.9796 \\
\hline
\end{tabular}

quired to approximate the surface charge density on the strips is much higher than the number of basis functions required to accurately approximate the $x$-component of the slot electric field.

As a final numerical example, we have computed the parameters of the six conductors geometry shown in Table IV. The horizontal symmetry plane $\left(\mathrm{AA}^{\prime}\right)$ is considered first an electric wall and then a magnetic wall in order to exploit the symmetry. Accuracy is set to four decimal figures for the normalized capacitance coefficients. This accuracy is achieved by using four basis functions at the $0.3 \mathrm{~mm}$ slots and three basis functions at the $0.1 \mathrm{~mm}$ and $0.2 \mathrm{~mm}$ slots. Total CPU time was 0.7 seconds on a $\mathrm{PC} / 486 / 33 \mathrm{MHz}$.

\section{CONCLUSION}

In this paper we have presented a technique to deal with the quasi-static analysis of multiconductor planar structures belonging to the family of coplanar waveguides. The method is based on the efficient solution of an integral equation for the electric field existing at the slots. Efficiency is achieved by means of analytical preprocessing of numerical series. The computer programs developed on the basis of this method are extremely accurate and numerically stable. In addition, CPU times are short enough to consider these programs useful in the frame of a computer aided design system. The electric field and surface charge density can be also computed with extreme accuracy.

\section{APPENDIX}

Two alternative formulations-which we have called spectral and spatial domain formulations respectively-have been used for the quasi-analytical determination of the asymptotic tails $S_{p, i}^{q, j}$.

\section{A. Spectral Domain Computation}

The computation of $S_{p, i}^{q, j}$ in (22) implies the addition of slowly convergent trigonometrical series of the following type:

$$
\left(S_{p, i}^{q, j}\right)^{\prime}=\sum_{n=1}^{\infty} \frac{J_{p}\left(n d_{i}\right) J_{q}\left(n d_{j}\right)}{n} \begin{cases}\cos \left(n c_{i j}^{ \pm}\right) & \text {if } p+q \text { even } \\ \sin \left(n c_{i j}^{ \pm}\right) & \text {if } p+q \text { odd }\end{cases}
$$

where $d_{i}=\frac{\pi s_{i}}{2 a}$ and $c_{i j}^{ \pm}=\frac{\pi}{a}\left(x_{c j} \pm x_{c i}\right)$.

These series have already appeared in the analysis of a multistrip configuration $[17,(8)]$. The residui calculus technique may be used to convert (23) into a much more quickly convergent power series (see [17] for more details)

$$
\begin{aligned}
& \left(S_{p, i}^{q, j}\right)^{\prime}=\left\{\begin{array}{c}
(-1)^{\frac{p+q}{2}} \\
(-1)^{\frac{p+q-1}{2}}
\end{array}\right\} \frac{\left(d_{j} / d_{i}\right)^{q}}{\Gamma(q+1)} \sum_{k=0}^{\infty}\left(\frac{d_{i}}{2}\right)^{p+q+2 k} \\
& \times \frac{F\left[-k,-p-k ; q+1 ;\left(d_{j} / d_{i}\right)^{2}\right]}{\Gamma(k+1) \Gamma(p+k+1)} \\
& \times \int_{0}^{\infty} d y \frac{y^{p+q+2 k-1}}{\sinh (\pi y)} \text {. } \\
& \times\left\{\begin{array}{llll}
\cosh \left[\left(\pi-c_{i j}^{ \pm}\right) y\right] & ; & p+q & \text { even } \\
\sinh \left[\left(\pi-c_{i j}^{ \pm}\right) y\right] & ; & p+q & \text { odd }
\end{array}\right.
\end{aligned}
$$

where $F$ is the hypergeometric function, and $\Gamma$, the gamma function. The hypergeometric function $F[-k,-p-k ; q+$ $\left.1 ;\left(d_{j} / d_{i}\right)^{2}\right]$ is a $k$-degree polynomial in $\left(d_{j} / d_{i}\right)^{2}$

$$
\begin{aligned}
& F\left[-k,-p-k ; q+1 ;\left(d_{j} / d_{i}\right)^{2}\right] \\
& =\sum_{n=0}^{k} \frac{\Gamma(k+1) \Gamma(p+k+1) \Gamma(q+1)\left(d_{j} / d_{i}\right)^{2 n}}{\Gamma(k-n+1) \Gamma(p+k-n+1) \Gamma(q+n+1) \Gamma(n+1)}
\end{aligned}
$$

Two alternative closed form expressions are known for the integrals appearing in (24)

$$
\begin{aligned}
& \int_{0}^{\infty} d y \frac{y^{\mu-1}}{\sinh (\pi y)}\left\{\begin{array}{l}
\cosh (\beta y) \\
\sinh (\beta y)
\end{array}\right\} \\
& =\left\{\begin{array}{l}
\frac{1}{2} \frac{d^{\mu-1}}{d \beta^{\mu-1}} \tan (\beta / 2) \\
\frac{\Gamma(\mu)}{(2 \pi)^{\mu}}\left\{\zeta\left[\mu, \frac{1}{2}\left(1-\frac{\beta}{\pi}\right)\right] \pm \zeta\left[\mu, \frac{1}{2}\left(1+\frac{\beta}{\pi}\right)\right]\right\}
\end{array}\right.
\end{aligned}
$$

where $\mu=p+q+2 k, \beta=\pi-c_{i j}^{ \pm}$, and $\zeta$ is the Riemann's zeta function. The first expression in (26) is used for the first few terms of the $k$-series. This suffices for most cases, but if larger values of $k$ are needed, the second expression in (26) provides an alternative quick solution.

The case $p=q=0$ requires a slightly different treatment

$$
\begin{aligned}
\left(S_{0, i}^{0, j}\right)^{\prime}=\sum_{k=1}^{\infty} & \frac{\Gamma(2 k+1) F\left[-k,-k ; 1 ;\left(d_{j} / d_{i}\right)^{2}\right]}{2 k \Gamma^{2}(k+1)}\left(\frac{d_{i}}{4 \pi}\right)^{2 k} \\
& \times\left\{\zeta\left[2 k, \frac{c_{i j}^{ \pm}}{2 \pi}\right]+\zeta\left[2 k, 1-\frac{c_{i j}^{ \pm}}{2 \pi}\right]\right\} \\
& -\ln \left[\sin \left(\frac{c_{i j}^{ \pm}}{2}\right)\right]-\ln (2) .
\end{aligned}
$$


Note the necessary presence of the integration constant, $\ln (2)$, which was not calculated in [17] because in that case it canceled out when the definitive $S_{0, i}^{0, j}$ was computed.

\section{B. Spatial Domain Computation}

Parseval and convolution theorems provide a second efficient alternative to compute $S_{p, i}^{q, j}$ from the quasi-analytical integration of its spatial counterparts

$$
\begin{aligned}
\frac{2}{a} S_{p, i}^{q, j}= & \int_{x_{c i}-s_{i} / 2}^{x_{i i i}+s_{i} / 2} d x \frac{2}{\pi s_{i}} \frac{T_{p}\left(\frac{x-x_{c i}}{s_{i} / 2}\right)}{\sqrt{1-\left(\frac{x-x_{c i}}{s_{i} / 2}\right)^{2}}} \\
& \times \int_{x_{c j}-s_{j} / 2}^{x_{c j}+s_{j} / 2} d x^{\prime} \frac{2}{\pi s_{j}} \frac{T_{q}\left(\frac{x^{\prime}-x_{c j}}{s_{j} / 2}\right)}{\sqrt{1-\left(\frac{x^{\prime}-x_{c j}}{s_{j} / 2}\right)^{2}}} \mathcal{L}_{a s}\left(x, x^{\prime}\right)
\end{aligned}
$$

where

$$
\begin{aligned}
\mathcal{L}_{a s}\left(x, x^{\prime}\right) & =\frac{2}{a} \sum_{n=1}^{\infty} \widetilde{\mathcal{L}}_{a s}\left(\alpha_{n}\right) \cos \left(\alpha_{n} x^{\prime}\right) \cos \left(\alpha_{n} x\right) \\
& =-\frac{2 \bar{\epsilon}}{\pi} \operatorname{In}\left\{4 \sin \left[\frac{\pi}{2 a}\left|x-x^{\prime}\right|\right] \sin \left[\frac{\pi}{2 a}\left(x+x^{\prime}\right)\right]\right\}
\end{aligned}
$$

The square root in the denominator of the integrands in (28) makes the Gauss-Chebyshev quadrature formula to be specially suitable for the computation of these integrals. However, direct Gauss-Chebyshev summation of the convolutions is not advisable because of the logarithmic singularity of $\mathcal{L}_{a s}\left(x, x^{\prime}\right)$ in $x=x^{\prime}$. Therefore, a previous extraction and separate integration of this singularity is very useful to increase the efficiency of the quadratures. When multistrip configurations are analyzed [17], the eventual proximity between the strips and the lateral walls introduces quasi-singular behavior of the integrand for $x+x^{\prime} \rightarrow 0$ or $x+x^{\prime} \rightarrow 2 a$. In coplanar waveguide-type configurations there is no such possibility as a consequence of the presence of the two lateral grounded fins. Consequently, the "singular part," $S\left(x, x^{\prime}\right)$, of $\mathcal{L}_{a s}\left(x, x^{\prime}\right)$ should be defined as:

$$
S\left(x, x^{\prime}\right)=-\frac{2 \bar{\epsilon}}{\pi} \ln \left|x-x^{\prime}\right| .
$$

If the kernel of (28) is split into the two following parts:

$$
\mathcal{L}_{a s}\left(x, x^{\prime}\right)=\left[\mathcal{L}_{a s}\left(x, x^{\prime}\right)-S\left(x, x^{\prime}\right)\right]+S\left(x, x^{\prime}\right)
$$

the contribution of the first term (very smooth function) to the convolution integrals is computed with a low order GaussChebyshev quadrature, and the contribution of the second term (singular part) can be analytically evaluated. Let $I_{i, j}[q ; x]$ be the convolution integral of the "singular part" except a constant factor

$$
\begin{gathered}
I_{i, j}[q ; x]=\int_{x_{c j}-s_{j} / 2}^{x_{c j}+s_{j} / 2} d x^{\prime} \frac{2}{\pi s_{j}} \frac{T_{q}\left(\frac{x^{\prime}-x_{c j}}{s_{j} / 2}\right)}{\sqrt{1-\left(\frac{x^{\prime}-x_{c j}}{s_{j} / 2}\right)^{2}}} \ln \left|x-x^{\prime}\right| \\
x_{c i}-s_{i} / 2 \leq x \leq x_{c i}+s_{i} / 2
\end{gathered}
$$

then, it can be demonstrated that for $i \neq j$

$$
\begin{aligned}
& I_{i, j}[q ; x] \\
& =\left\{\begin{array}{cl}
-\frac{1}{q}\left[t_{i j}(x)-\operatorname{sgn}\left(t_{i j}(x)\right) \sqrt{\left(t_{i j}(x)\right)^{2}-1}\right]^{q} & \text { if } q \geq 1 \\
\ln \left[\frac{s_{j}}{4}\left(\left|t_{i j}(x)\right|+\sqrt{\left(t_{i j}(x)\right)^{2}-1}\right)\right] & \text { if } q=0
\end{array}\right.
\end{aligned}
$$

$$
t_{i j}(x)=\frac{x-x_{c j}}{s_{j} / 2} ; \quad x_{c i}-\frac{s_{i}}{2} \leq x \leq x_{c i}+\frac{s_{i}}{2}
$$

where $\operatorname{sgn}(\cdot)$ is the sign function, and for $i=j$

$$
I_{i, i}[q ; x]= \begin{cases}-\frac{1}{q} T_{q}\left(t_{i i}\right) & \text { if } q \geq 1 \\ \ln \left(s_{i} / 4\right) & \text { if } q=0 .\end{cases}
$$

The last step for the computation of (28) is to carry out the inner products. Closed form expressions have been found only for the case $i=j$

$$
\begin{gathered}
\int_{x_{c i}-s_{i} / 2}^{x_{c i}+s_{i} / 2} d x \frac{2}{\pi s_{i}} \frac{T_{p}\left(\frac{x-x_{c i}}{s_{i} / 2}\right)}{\sqrt{1-\left(\frac{x-x_{c i}}{s_{i} / 2}\right)^{2}}} I_{i, i}[q ; x] \\
\quad=\left\{\begin{array}{lll}
-1 / 2 p & \text { if } p=q \neq 0 \\
0 & \text { if } p \neq q \\
\ln \left(s_{i} / 4\right) & \text { if } p=q=0 .
\end{array}\right.
\end{gathered}
$$

The rest of the inner products have been numerically evaluated by low order Gauss-Chebyshev quadratures.

\section{REFERENCES}

[1] C. P. Wen, "Coplanar-waveguide directional couplers," IEEE Trans. Microwave Theory Tech., vol. MTT-18, pp. 318-322, June 1970.

[2] R. A. Pucel, "Design considerations for monolithic microwave circuits," IEEE Trans. Microwave Theory Tech., vol. MTT-29, pp. 513-534, June 1981.

[3] R. W. Jackson, "Considerations in the use of coplanar waveguide for millimeter-wave integrated circuits," IEEE Trans. Microwave Theory Tech., vol. MTT-34, pp. 1450-1456, Dec. 1986.

[4] A. A. Omar and Y. L. Chow, "A solution of coplanar waveguide with air-bridges using complex images," IEEE Trans. Microwave Theory Tech., vol. 40, pp. 2070-2077, Nov. 1992.

[5] N. I. Dib, G. E. Ponchak, and L. P. B. Katehi, "A theoretical and experimental study of coplanar waveguide shunt stubs," IEEE Trans. Microwave Theory Tech., vol. 41, pp. 38-44, Jan. 1993.

[6] M. Gillick, I. D. Robertson, and J. S. Joshi, "Direct analytical solution for the electric field distribution at the conductor surfaces of coplanar waveguides," IEEE Trans. Microwave Theory Tech., vol. 41, pp. 129-135, Jan. 1993.

[7] G. Mazé-Merceur, S. Tedjini, and J.-L. Bonnefoy, "Analysis of a CPW on electric and magnetic biaxial substrate," IEEE Trans. Microwave Theory Tech., vol. 41, pp. 457-461, Mar. 1993.

[8] G. Ghione and C. U. Naldi, "Coplanar waveguides for mmic applications: effect of upper shielding, conductor backing, finite-extent ground planes, and line-to-line coupling," IEEE Trans. Microwave Theory Tech., vol. MTT-35, pp. 260-267, Mar. 1987.

[9] S. S. Bedair and I. Wolff, "Fast and accurate analytic formulas for calculating the parameters of a general broadside-coupled coplanar waveguide for (M)MIC applications," IEEE Trans. Microwave Theory Tech., vol. 37, pp. 843-850, May 1989.

[10] $\longrightarrow$, "Fast, accurate and simple approximate analytic formulas for calculating the parameters of supported coplanar waveguides for (M)MIC's," IEEE Trans. Microwave Theory Tech., vol. 40, pp. 41-48, Jan. 1992.

[11] E. Drake, F. Medina, and M. Horno, "Quasi-analytical static solution for the generalized boxed coplanar waveguide," Int. J. Microwave and Millimeter-Wave Computer-Aided Engineering, vol. 4, pp. 163-174, Apr. 1994.

[12] J.S. McLean and T. Itoh, "Analysis of a new configuration of coplanar stripline," IEEE Trans. Microwave Theory Tech., vol. 40, pp. 772-774, Apr. 1992. 
[13] T. Kitazawa, Y. Hayashi, and R. Mittra, "Asymmetrical coupled coplanar-type transmission lines with anisotropic substrates," IEE Proc., Microwaves, Optics Antennas, vol. 133, pt. H, pp. 265-270, Aug. 1986

[14] T. Kitazawa and T. Itoh, "Propagation characteristics of coplanar-type transmission lines with lossy media," IEEE Trans. Microwave Theory Tech., vol. 39, pp. 1694-1700, Oct. 1991.

[15] A. F. Molisch, A. R. Baghai-Wadji, and C. O. Schiebl, "On the application of the Wiener-Hopf technique to electrostatic field problems in interdigital transducers," IEEE Trans. Microwave Theory Tech., vol. 41, pp. 318-324, Feb. 1993.

[16] G. E. Howard, J. J. Yang, and Y. L. Chow, "A multipipe model of general strip transmission lines for rapid convergence of integral equation singularities," IEEE Trans. Microwave Theory Tech., vol. 40, pp. 628-636, Аpr. 1992.

[17] E. Drake, F. Medina, and M. Horno, "Improved quasi-TEM spectral domain analysis of boxed coplanar multiconductor microstrip lines" IEEE Trans. Microwave Theory Tech., vol. 41, pp. 260-267, Feb. 1993.

[18] - "Un análisis eficiente de líneas microtiras multiconductoras para PC's," Proc. of VII Symp. Nacional U.R.S.I., pp. 831-835, Málaga, Spain.

[19] M. Horno, F. L. Mesa, F. Medina, and R. Marqués, "Quasi-TEM analysis of multilayered, multiconductor coplanar structures with dielectric and magnetic anisotropy including substrate losses," IEEE Trans. Microwave Theory Tech., vol. 38, pp. 1059-1068, Aug. 1990.

[20] S. Haykin, Communication Systems. New York: Wiley, 1983.

[21] F. Medina and M. Horno, "Upper and lower bounds on mode capacitances for a large class of anisotropic multilayered microstriplike transmission lines," Proc. Inst. Elec. Eng. (Microwaves, Optic Antennas), vol. 132, no. 3, pp. 157-163, June 1985.

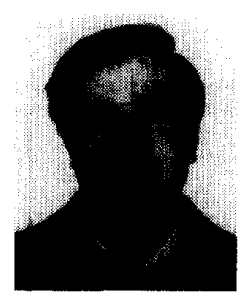

Enrique Drake was born September 4, 1966, in Montilla, Cárdoba, Spain. He received the Licenciado degree in physics from the University of Seville, Spain, in 1990 . He is currently following a Ph.D. program in Microwaves.

He is Assistant Professor at the Department of Applied Physics at the University of Seville since 1992. His research interest focus on the analysis of planar structures and multiconductor lines.

Francisco Medina, for a photograph and biography, see page 1631 of the September issue of this TRANSACTIONS.

Manuel Horno (M'75), for a photograph and biography, see page 432 of the March issue of this TRANSACTIONS. 